\title{
MEASURING AND FOSTERING ENGAGEMENT with Mental Health e-Coaches
}

PREPRINT

\author{
Zoraida Callejas, David Griol, Kawtar Benghazi, Manuel Noguera \\ Software Engineering Department, University of Granada, Spain \\ \{zoraida, dgriol, benghazi, mnoguera\}@ugr.es
}

Gérard Chollet

Intelligent Voice, London, UK

gerard.chollet@intelligentvoice.com

María Inés Torres

Dept. of Electrical and Electronics, University of the Basque Country, Leioa, Spain

manes.torres@ehu. eus

Anna Esposito

University of Campania Luigi Vanvitelli, Caserta, Italy

iiass . annaesp@tin.it

This is a pre-print version of the paper:

Zoraida Callejas, David Griol, Kawtar Benghazi, Manuel Noguera, Gerard Chollet, María Inés Torres, and Anna Esposito. 2020. Measuring and Fostering Engagement with Mental Health e-Coaches. In Companion Publication of the 2020 International Conference on Multimodal Interaction (ICMI '20 Companion), October 25-29, 2020, Virtual event, Netherlands. ACM, NewYork, NY, USA, 5 pages. https://doi.org/10.1145/3395035.342531

This preprint follows ACM policy: "Authors who publish with ACM have the freedom to post peer-reviewed pre-print versions of their papers to personal websites and institutional repositories. They can add a single-click link to their final published papers, and re-use any portion of their published work with the inclusion of a citation and DOI link. Authors can also post on any repository legally mandated by the agency funding the research on which the work is based, and on any non-commercial repository or aggregation that does not duplicate ACM tables of contents/substantially duplicate an ACM-copyrighted volume or issue" (https://authors.acm.org/author-resources/author-rights.) 


\begin{abstract}
Mental health e-coaches and technology-delivered services are showing considerable benefits to foster mental health literacy, monitor symptoms, favour self-management of different mental health conditions and scaffold positive behaviours.

However, adherence to these systems is usually low and generally declines over time. There exists a recent body of work addressing engagement with mental health technology with the aim to understand the factors that influence sustained use and inform the design of systems that are able to generate sufficient engagement to attain their expected results.

This paper explores the different facets of engagement in mental health e-coaches, including aspects related to the estimation of system use from log data, effective engagement, user experience, motivation, incentives, user expectations, peer support and the specific challenges of technologies addressed to mental health.
\end{abstract}

Keywords Multimodal interaction · virtual coach · e-coach · distributed interface $\cdot$ ubiquitous computing

\title{
1 Introduction
}

Meeting the demands for prevention and treatment of common mental health disorders constitutes a major health challenge [1]. However, access to mental health services encounters numerous barriers such as long waiting lists, difficulties to access services in remote areas, access to 24/7 services, cost, perceived stigma and lack of mental health literacy.

Some of these difficulties can be addressed by combining traditional services with the use of information and communication technology (ICT) to support and improve mental health conditions and care. Mental health e-coaches are systems which objective is to promote a good mental health of the user, usually delivering tailored interventions, activities and multimedia content and assessing the state of the user through periodic screening and automated symptom questionnaires.

As described in [2], e-mental health covers a wide range of applications including screening, health promotion, early intervention, treatment, and relapse prevention. It is also very valuable for self-management of mental health, allowing to self-track thoughts, behaviours, and emotions which is specially useful in chronic conditions [3]. In addition, facilitating the widespread use of mental health e-coaches can enable professionals to attain better evidence-based care, engage patients more actively and establish a communication line that can be active after treatment [4].

Smartphone based coaches also provide sensor data that serve as predictors of mental health conditions and relapse, helping to understand patients, trigger timely intervention and deliver tailored therapy [5].

To unveil their full potential, mental health e-coaches must be used with a certain frequency and over a period of time that makes it possible to achieve and evaluate their potential benefits. In this context, engagement may be defined as "starting and continuing to work with the intervention" [6]. When the work is following exactly how the intervention was designed/prescribed, it is also possible to use the term adherence. On the other hand, attrition, abandonment and dropout describe the situation when the user stops participating in the intervention prior the planned ending.

Despite the potential of mental health e-coaches, consistent evidence from multiple studies suggests that a significant number of people may be unwilling to engage with ICT delivered interventions and for those who use them there is usually a decline in use over time, resulting in limited engagement, high attrition and low adherence rates [7, 8, 6]. This does not only limit the reach of the tool and its benefits, but also affects the validity of the findings derived from their use [9].

We present an overview on how engagement is understood, represented and measured in artificial coaches for mental health. The rest of the paper is organised as follows: section 2 presents different visions on engagement and how it can be measured in terms of objective system use and subjective user experience; section 3 explores ways in which engagement can be fostered by increasing intrinsic and extrinsic motivation; section 4 addresses the barriers to engagement that are specific for mental health technologies and finally section 5 presents the conclusions and indicates future work guidelines. 


\section{Measuring engagement}

ICT-based mental health coaching provides the opportunity to estimate user engagement objectively with quantitative measures of system use. In addition to quantitative measures, engagement can also be assessed in terms of the user's subjective vision of their own involvement, which may include user's thoughts and feelings during system use.

\subsection{Quantitative assessment}

Engagement can be computed from log data that is automatically acquired and stored by the system. For example the number of logins or number of daily active users, user traffic (e.g. website), number of activities/interventions completed, number of multimedia played / documents read or accessed, duration of sessions / minutes of use, frequency of sessions, or user retention after a month.

From these basic variables, more complex measures can be obtained considering the total duration of the intervention. This way, Arnold et al. [7] compute depth of use as the total use (e.g. number of activities completed) over the intervention period, and breadth of use as the variation in use distinguishing between different types of activities, for example active - which require more user involvement (e.g. completing an exercise) - and passive activities - e.g. watching a video.

Also the level of use can be compared to the intended usage. As discussed in [10], approaches to measuring adherence vary due to the diversity in the purpose and design of digital interventions.

Interestingly, not always more use is better. Some studies indicate that more logins and duration is positive, while others found they do not affect the outcomes.

On the one hand, system usage duration and frequency are not only a measure of engagement, but can be indicators of relapse. For example the results in [11] suggest that higher phone usage duration and frequency is correlated with higher levels of depressive symptom severity.

In contrast, [8] uses the expression "effective engagement" or efficiency, when there is sufficient engagement to achieve the intended outcomes. From this perspective, a low retention rate of the users may not be negative, but an indicative that they have acquired the desired skills or knowledge.

As discussed in [12] this variability may be due to the fact that after a certain minimal amount of program use, the most significant aspect to attain a better result is the content provided by the system.

Log data can also be used to monitor the performance of the users. However, performance monitoring can be damaging if it is understood as surveillance and can be demoralising and lead to attrition [13]. This is why it is fundamental to explain clearly how and why monitoring will take place to mitigate its negative effects (see Section 3 ).

Other objective measures are used to try to assess the number of users engaged in a particular mental health application. However, the high attrition rates encountered in the literature suggest that parameters such as the number of app installs (e.g. in Google Play) do not offer an adequate estimation of the proportion of users who are actively engaged in using the app [8].

\subsection{Qualitative user experience}

Qualitative assessments of engagement are usually performed by means of interviews and self-reports. The design of the protocol to conduct this studies is crucial to obtain valid results. For example, to perform their user experience evaluation, Mohr et al. [14] took into consideration two important aspects: i) the interviews were administered by trained clinical evaluators who were unaware of the usage data; ii) the self-reported outcomes were administered via a website different from the program under evaluation.

In [4], Chen et al. collected participants' SMS conversations with coaches about their experience. In this case, the evaluation of the information retrieved is not done entirely in a qualitative fashion, but they use a simple natural language processing protocol to extract salient concepts related to experience, usage, improvement, usability and other aspects.

An interesting aspect that affects the studies of user experience is related to the enrolment method. As discussed by [8], scientists may unintentionally recruit people who are much more likely to engage with the technology than the general population who could potentially download and try it. In [4], users could be engage in a suite of up to 14 apps and could start and stop using any app at any time, the difficulty encountered by the authors was to characterise user interactions and engagement in this challenging setting. 
As argued in [15], when confronted with recruitment challenges (e.g. finding enough subjects with a particular conditions), there may be the tendency to search until finding enough people willing to use the technology, which undermines the generalizability of the findings. The authors indicate that recruitment challenges should instead be considered indications of failures in the design of the system or the underlying strategy.

\section{Fostering engagement}

Motivation of the users to employ the e-coach and towards the achievement of its potential benefits is key for engagement. Motivation can be intrinsic because the user has assumed the skills and effort required to achieve their goals, or external motivating factors. According to [13], to foster internal motivation to use e-health applications, they must be engaging, interesting and salient to the user. As highlighted in [7], internal motivation results in enhanced and maintained engagement, whereas extrinsic motivators do not support long term engagement. The authors found that when tools were recommended by family or clinicians, the users only used them occasionally.

It is essential that users understand and agree with the underlying rationale and objectives of the mental health e-coach to foster motivation and facilitate engagement. Mohr et al. build on accountability theory to distinguish two types of expectations: outcome accountability and process accountability [13]. The first can be defined in terms of the results achieved (e.g. a decrease in depression severity), the latter is related to objective usage measures such as the ones described in Section 2.1. Relying mainly in process accountability with rigid adherence goals that are not related to patients larger goals and values may be detrimental [13], and thus goal setting must be designed carefully.

Another way of fostering motivation is to use incentives and rewards in exchange for engaging in mental health e-coaching, specially for research purposes. With respect to tangible rewards, some studies have shown that small incentives can have a very positive effect on initial adoption [16], while other aspects (e.g. the salience of feedback) can be more important for sustained use. However, [13] warns about the pernicious effect of tangible rewards in intrinsic motivation, specially when they are perceived by the user as a cue that they are perceived as lacking intrinsic motivation or as a means of control. The experimental results in [9] indicate that engagement was lower when participants were offered monetary compensation and were reminded to engage in the intervention.

On the contrary, verbal rewards enhance intrinsic motivation, specially when they reassures competence as long as they are not offered in a controlling manner [13]. Nevertheless, the authors emphasise it is important to make clear that a failure to meet the expectations is an opportunity for self-reflection and growth without negative consequences.

\subsection{Relational factors}

There are different types of mental health applications, those that provide autonomous automated advice and are not operated by a professional at the other end, and those that either make it possible for professionals to control the intervention or directly serve as a way of communicating users with professionals or peers. "Unguided" applications reduce the costs and favour access to services, but they have limited power to engage users in the activities [8], as they compete with their daily life activities and require a high motivation.

Relational factors have been pointed in the literature as an important source of motivation and engagement. For example, [15] indicates that substantive mental health benefits are more consistently achieved in the context of human support. Their vision is that mental health technologies should not be considered stand-alone products, but rather "technology-enabled services" that should fit in the context of mental health services. Under this vision, they are services that are supported by technologies rather than as human-supported technologies and it is thus fundamental to question what is the role of human support, which may vary from just sustaining engagement to support goals, gain insight, give feedback and positive reinforcement, etc.

Human support can also come from peers living with similar mental health challenges. O'Leary et al. found that people with mental illnesses many times go online to find peers who can provide information, emotional support, and advice [17]. The authors show that, although online peer support can bring benefits, it may also be a source of negative experiences, which include unsupportive members, negative content, and problems related with the stigma that is many times associated to mental illness. These drawbacks could be diminished if they are taken into consideration during design, particularly when engaging peer supporters in co-creation.

In particular, [13] discusses that although automated efforts to foster engagement such as email reminders can improve adherence, human support boots engagement to a greater degree.

Another interesting aspect is the role of researchers. For example, [18] found that the degree of engagement of their users with the app depended on the rapport with the researcher facilitating delivery. 
In our previous research (to be published in the proceedings of IWSDS 2020 https://www. iwsds.tech/), people with mild depression and anxiety perceive the apps as useful and think they can facilitate seeking help at times when they would not do so with another person, either because of the time (e.g. late night) or because they feel they can be a burden for that person (e.g. family members).

\section{Barriers to engagement}

E-coaches addressed at mental health must take into consideration certain specific challenges that are not present or have a milder impact in other health coaches. Arnold et al. [19] identified challenges to use their system (a web-based psycho-social intervention for psychosis), which included some very specific of mental health such as: fluctuating levels of wellness or "not wanting to dig too deep".

Mental health self management with e-coaches generally entails some type of symptom reporting, which despite its benefits, may have some risks. First, daily reporting of symptoms may be perceived as repetitive or even unnecessary. Secondly, it can be discouraging to regularly remind users about their mental illness, specially at times when they felt alright [19, 10].

Murnane et al. [3] warn that providing feedback about negative emotional and behavioural patterns could itself trigger or exacerbate symptoms. This can also threaten the fragile view that some users may have of themselves [18]. Aref-Adib et al. [20] also found that their users sometimes felt fear and anxiety about what they read in the resources provided, specially about the side-effects of anti-psychotic medication.

On the contrary, relying on implicit monitoring using sensors and other technology transparent to the user, potentially undermines the agency and empowerment related to explicit self-monitoring [3]. Thus, there is complicated balance that the designers of mental health e-coaches must achieve considering these different aspects.

In general, when in low mood or unwell the motivation to engage with e-health applications decreases and several studies report that mental health symptoms interfere with user engagement [19]. Eisner et al. found that the extent of such interference varies [18].

Different authors agree in the importance of understanding the factors associated with engagement for specific mental health illnesses. For example, [7] found for psychosis generally low computer literacy and limited Internet access secondary to low socio-economic status.

Hatch et al. [21] provide a survey conducted with a panel of 40 experts about digital health tools for patients with serious mental illness. The experts interviewed did not consider diagnoses of schizophrenia, bipolar disorder, or major depressive disorder likely to influence the ability to use a digital health tool. However, some of their symptoms were considered to have an effect. These included: severe positive, negative, disorganized, and neurocognitive symptoms; acute substance abuse; agitation or aggression; low energy; and tolerance for frustration.

On aspects not related to their mental illness, the main aspect highlighted was the patient's interest and their positive expectations about the use of the tool. Negative past experience with treatment, limited insight into their illness, and a high level of chaos or disorganization in patient's lives were rated as likely to make it more difficult for patients to engage with or use digital health tools.

Perceived ability and self-efficacy has been also a relevant factor encountered in the literature. For example, [22] presents a study of telephone-based coaching for chronic disease health risk behaviours engaging users with and without a mental health conditions. Their results show that participants with a mental health condition experienced less confidence in their ability to engage in behaviour change, which may inhibit engagement.

Another issue to be considered is the lack of time that some collectives may have due to the high number of medical and mental health appointments as well as time spent attending to other health difficulties [23].

\section{Conclusions and future work}

E-coaches offer great potential to support and improve mental health conditions and mental health care. However, in order to inform more effective design and implementation as well as to evaluate the efficacy of e-coaches interventions, it is crucial to identify and understand the dimensions and predictors that promote engagement and continued use [7, 24].

This paper has presented a review of some of the main aspects related to engagement in relation with mental health e-coaches. Evidence suggests that engagement must be considered not only in terms of frequency of use, but also 
gaining insight about user experience and user self-selected activity to enact positive behaviour and favour successful self-management.

Low adherence can be an indicator of relapse, but at the same time may be the result of users successfully self-managing their condition and needing less support by the e-coach.

Monetary compensation and automated reminders may help for initial engagement but their efficacy decreases and they may be even detrimental to encourage users' agency. Instead, autonomous intrinsic motivation should be promoted by establishing clear, process-oriented expectations and clarifying the system objectives, which must be shared by the users. Peer support and therapeutic alliance play an important role to this respect.

Continuous monitoring can be beneficial to foster self-reflection, for symptom alert with at-risk populations, and to maintain interest, but it can also be a constant reminder of mental health problems and a potential burden, so it must designed with flexibility.

In general, to optimise the development of mental health e-coaches it is important to tailor the system to accommodate them to the circumstances and peculiarities of individuals and favor person-to-person contact both at design time (with co-creation panels) as well through the technology. Adequate training and reassuring confidence of the users in their ability to use the technology are also key for adoption and engagement.

For future work, in the context of the MENHIR project (https://menhir-project.eu which aim is to promote mental health through conversational technologies, we plan to investigate which aspects of spoken conversation with e-coaches (including content, wording, prosody, voice and conversational features) lead to sustained engagement. We will propose a set of good practices and validate it with a panel of researchers, ICT and psychology experts, mental health providers and their clients.

\section{Acknowledgements}

This research has received funding from the European Union's Horizon 2020 research and innovation programme under grant agreement No 823907 (MENHIR project https://menhir-project.eu) and grant agreement No 769872 (EMPATHIC project http://www.empathic-project.eu).

\section{References}

[1] Anna Paldam Folker, Kim Mathiasen, Sigurd Mørk Lauridsen, Ellen Stenderup, Els Dozeman, and Marie Paldam Folker. Implementing internet-delivered cognitive behavior therapy for common mental health disorders: A comparative case study of implementation challenges perceived by therapists and managers in five European internet services. Internet Interventions, 11:60-70, March 2018.

[2] Heleen Riper, Gerhard Andersson, Helen Christensen, Pim Cuijpers, Alfred Lange, and Gunther Eysenbach. Theme Issue on E-Mental Health: A Growing Field in Internet Research. Journal of Medical Internet Research, 12(5):e74, 2010.

[3] Elizabeth L. Murnane, Mark Matthews, and Geri Gay. Opportunities for technology in the self-management of mental health. In Proceedings of the 18th International Conference on Human-Computer Interaction with Mobile Devices and Services Adjunct - MobileHCI '16, pages 1093-1096, Florence, Italy, 2016. ACM Press.

[4] Annie T. Chen, Shuyang Wu, Kathryn N. Tomasino, Emily G. Lattie, and David C. Mohr. A multi-faceted approach to characterizing user behavior and experience in a digital mental health intervention. Journal of Biomedical Informatics, 94:103187, June 2019.

[5] Simon D'Alfonso, Nathaniel Carpenter, and Mario Alvarez-Jimenez. Making the MOST out of smartphone opportunities for mental health. In Proceedings of the 30th Australian Conference on Computer-Human Interaction, OzCHI '18, pages 577-581, New York, NY, USA, December 2018. Association for Computing Machinery.

[6] Kate Cavanagh. Turn on, tune in and (don't) drop out: Engagement, adherence, attrition, and alliance with internet-based interventions. In Oxford guide to low intensity CBT interventions, Oxford guides in cognitive behavioural therapy, pages 227-233. Oxford University Press, New York, NY, US, 2010.

[7] Chelsea Arnold, Kristi-Ann Villagonzalo, Denny Meyer, John Farhall, Fiona Foley, Michael Kyrios, and Neil Thomas. Predicting engagement with an online psychosocial intervention for psychosis: Exploring individualand intervention-level predictors. Internet Interventions, 18:100266, December 2019. 
[8] Amit Baumel, Frederick Muench, Stav Edan, and John M Kane. Objective User Engagement With Mental Health Apps: Systematic Search and Panel-Based Usage Analysis. Journal of Medical Internet Research, 21(9), September 2019.

[9] Jake Linardon and Matthew Fuller-Tyszkiewicz. Attrition and adherence in smartphone-delivered interventions for mental health problems: A systematic and meta-analytic review. Journal of Consulting and Clinical Psychology, 88(1):1-13, 2020. Place: US Publisher: American Psychological Association.

[10] Marie-Therése Crafoord, Maria Fjell, Kay Sundberg, Marie Nilsson, and Ann Langius-Eklöf. Engagement in an Interactive App for Symptom Self-Management during Treatment in Patients With Breast or Prostate Cancer: Mixed Methods Study. Journal of Medical Internet Research, 22(8), 2020.

[11] Sohrab Saeb, Mi Zhang, Christopher J. Karr, Stephen M. Schueller, Marya E. Corden, Konrad P. Kording, and David C. Mohr. Mobile Phone Sensor Correlates of Depressive Symptom Severity in Daily-Life Behavior: An Exploratory Study. Journal of Medical Internet Research, 17(7):e175, 2015.

[12] Robin M. Lally, Kevin Kupzyk, Steve Gallo, and Donna Berry. Use of an Unguided, Web-Based Distress Self-Management Program After Breast Cancer Diagnosis: Sub-Analysis of CaringGuidance Pilot Study. Journal of Medical Internet Research, 22(7):e19734, 2020.

[13] David Mohr, Pim Cuijpers, and Kenneth Lehman. Supportive Accountability: A Model for Providing Human Support to Enhance Adherence to eHealth Interventions. Journal of Medical Internet Research, 13(1), 2011.

[14] David C. Mohr, Jennifer Duffecy, Ling Jin, Evette J. Ludman, Adam Lewis, Mark Begale, and Martin McCarthy Jr. Multimodal E-Mental Health Treatment for Depression: A Feasibility Trial. Journal of Medical Internet Research, 12(5), 2010.

[15] David C. Mohr, Ken R. Weingardt, Madhu Reddy, and Stephen M. Schueller. Three Problems With Current Digital Mental Health Research . . . and Three Things We Can Do About Them. Psychiatric Services, 68(5):427-429, April 2017.

[16] Jacob Brower, Monica C. LaBarge, Lauren White, and Marc S. Mitchell. Examining Responsiveness to an Incentive-Based Mobile Health App: Longitudinal Observational Study. Journal of Medical Internet Research, 22(8), 2020.

[17] Kathleen O’Leary, Arpita Bhattacharya, Sean A. Munson, Jacob O. Wobbrock, and Wanda Pratt. Design Opportunities for Mental Health Peer Support Technologies. In Proceedings of the 2017 ACM Conference on Computer Supported Cooperative Work and Social Computing, pages 1470-1484, Portland Oregon USA, February 2017. ACM.

[18] Emily Eisner, Richard James Drake, Natalie Berry, Christine Barrowclough, Richard Emsley, Matthew Machin, and Sandra Bucci. Development and Long-Term Acceptability of ExPRESS, a Mobile Phone App to Monitor Basic Symptoms and Early Signs of Psychosis Relapse. JMIR mHealth and uHealth, 7(3), 2019.

[19] Chelsea Arnold, Anne Williams, and Neil Thomas. Engaging With a Web-Based Psychosocial Intervention for Psychosis: Qualitative Study of User Experiences. JMIR Mental Health, 7(6), 2020.

[20] Golnar Aref-Adib, Puffin O’Hanlon, Kate Fullarton, Nicola Morant, Andrew Sommerlad, Sonia Johnson, and David Osborn. A qualitative study of online mental health information seeking behaviour by those with psychosis. BMC Psychiatry, 16(1):232, July 2016.

[21] Ainslie Hatch, Julia E Hoffman, Ruth Ross, and John P Docherty. Expert Consensus Survey on Digital Health Tools for Patients With Serious Mental Illness: Optimizing for User Characteristics and User Support. JMIR Mental Health, 5(2), June 2018.

[22] Tegan Bradley, Kate Bartlem, Elizabeth Campbell, Paula Wye, Chris Rissel, Kate Reid, Timothy Regan, Jacqueline Bailey, and Jenny Bowman. Characteristics of participants utilising a telephone-based coaching service for chronic disease health risk behaviours: A retrospective examination comparing those with and without a mental health condition. Preventive Medicine Reports, 19, 2020.

[23] Eric DA Hermes, Jeremy Merrel, Ashley Clayton, Christa Morris, and Michael Rowe. Computer-based self-help therapy: A qualitative analysis of attrition. Health Informatics Journal, 25:41-50, 2019.

[24] Emily A. Scherer, Dror Ben-Zeev, Zhigang Li, and John M. Kane. Analyzing mHealth Engagement: Joint Models for Intensively Collected User Engagement Data. JMIR mHealth and uHealth, 5(1), 2017. 